\title{
LETTER
}

\section{Effect of body position and inclination in supine and prone position on respiratory mechanics in acute respiratory distress syndrome}

\author{
Mehdi Mezidi ${ }^{1,2}$ and Claude Guérin ${ }^{1,2,3,4,5^{*}}$ (]) \\ ๑ 2018 Springer-Verlag GmbH Germany, part of Springer Nature
}

\section{Introduction}

The mechanism by which prone position (PP) reduces mortality in moderate-to-severe acute respiratory distress syndrome (ARDS) patients as compared to supine (SP) [1] likely includes reduction/homogenization of lung stress/strain. The common accepted scenario is that this effect stems from an increase in chest wall elastance $(E s t, \mathrm{cw})$ in PP. However, whether, and to what extent, the angulation of the body may affect Est,cw is unclear. Our hypothesis is that thorax inclination significantly changes respiratory system mechanics and lung volume from SP to PP, as previously suggested in healthy humans [2] and anesthetized patients [3].

\section{Methods}

The present report is an ancillary study of a previous published work [4]. In ARDS patients receiving continuous infusion of sedative and neuromuscular blockade agents, lung and chest wall mechanics, end-expiratory lung volume (EELV), and transcutaneous oxygen saturation $\left(\mathrm{SpO}_{2}\right)$ were measured in the following order: $\mathrm{SP}$ $30^{\circ}$ head-up $\left(\mathrm{SP} 30^{\circ}\right)$, SP flat $\left(\mathrm{SP}^{\circ}\right)$, $\mathrm{PP}$ flat $\left(\mathrm{PP}^{\circ}\right)$, and $\mathrm{PP}$ $15^{\circ}$ head-up $\left(\mathrm{PP} 15^{\circ}\right)$. Each position was applied during $10 \mathrm{~min}$, at constant ventilator settings. Esophageal pressure (Pes) was measured via Nutrivent catheter (Sidam, Mirandola, Italy) and EELV by nitrogen washin-washout

*Correspondence: claude.querin@chu-lyon.fr

1 Service de Médecine Intensive-Réanimation, Hôpital de La Croix-Rousse, Hospices civils de Lyon, Lyon, France

Full author information is available at the end of the article technique from the ventilator. Complete methodology can be found in ESM. The primary outcome was Est,cw.

Data is expressed as mean (95\% confidence intervals) and compared by using analysis of variance for repeated measures with pairwise comparisons with the Holm test.

\section{Results}

Data was prospectively acquired in 24 consecutive ARDS patients (21 moderate and 3 severe). ARDS etiology was mainly pneumonia. Average tidal volume was 0.385 (0.357-0.414) L or $5.9(5.7-6.1) \mathrm{mL} / \mathrm{kg}$ predicted body weight), positive end-expiratory pressure (PEEP) 11 (1012) $\mathrm{cmH}_{2} \mathrm{O}$, and respiratory rate 31 (29-33) breaths/min.

Static end-expiratory Pes (PEEPtot,es) significantly increased from $\mathrm{SP} 30^{\circ}$ to $\mathrm{SP} 0^{\circ}$, then decreased at $\mathrm{PPO}^{\circ}$, and further declined at $\mathrm{PP} 15^{\circ}$ at which baseline value was returned (Table 1). Because plateau Pes exhibited similar changes as PEEPtot,es, both driving Pes and Est,cw decreased at $\mathrm{SPO}^{\circ}$, then increased and so went lung elastance across positions.

EELV significantly and continuously dropped from $\mathrm{SP} 30^{\circ}$ to $\mathrm{PP} 0^{\circ}$ and then increased but did not recover the $\mathrm{SP} 30^{\circ}$ level.

\section{Discussion}

This is the first study that systematically assesses respiratory mechanics in various inclinations and positions in the modern era of ARDS management. It confirms that Est,cw increases from SP to PP [5]. It discloses the significant role of body inclination. Indeed, the change in Est,cw with change in position is significant at $0^{\circ}$ inclination only. $\mathrm{PP} 15^{\circ}$ is associated with higher EELV and static 


$$
1
$$


end-expiratory transpulmonary pressure but higher lung driving pressure and elastance as compared to $\mathrm{PPO}^{\circ}$.

Two findings are worth noting. The first is the lower Est, $\mathrm{cw}$ in supine flat vs. supine $30^{\circ}$ and in prone flat vs. prone $15^{\circ}$. Gastric pressure actually decreased in supine flat as compared to supine $30^{\circ}$, resulting in less impact of the abdominal content onto the thorax. Furthermore, as a result of the EELV decrease in flat positions, the chest wall may have been moved to a more compliant part of its volume-pressure curve. Second, at the clinical standard for positioning (supine $30^{\circ}$ vs. prone $0^{\circ}$ ) elastances are the same. This finding emphasizes the importance of body angulation when dealing with respiratory mechanics during changing position.

This study is limited by both the short time of application and the lack of randomization of each position. However, it is the common way the procedure of proning is done in routine at the bedside, making the present results relevant in clinical practice.

To conclude, this study found that the body inclination may significantly affect respiratory mechanics, and hence should be taken into account in further clinical studies and in daily practice.

\section{Electronic supplementary materia}

The online version of this article (https://doi.org/10.1007/s00134-018-5493-1) contains supplementary material, which is available to authorized users.

\section{Author details}

${ }^{1}$ Service de Médecine Intensive-Réanimation, Hôpital de La Croix-Rousse, Hospices civils de Lyon, Lyon, France. ${ }^{2}$ Université de Lyon, Lyon, France.
${ }^{3}$ INSERM 955, Créteil, France. ${ }^{4}$ Service de Médecine Intensive-Réanimation, Grenoble, France. ${ }^{5}$ HP2 INSERM 1042, Grenoble, France.

Compliance with ethical standards

\section{Conflicts of interest}

The authors declare no conflict of interest.

Accepted: 3 December 2018

Published online: 7 December 2018

\section{References}

1. Guerin C, Reignier J, Richard JC, Beuret P, Gacouin A, Boulain T, Mercier E, Badet M, Mercat A, Baudin O, Clavel M, Chatellier D, Jaber S, Rosselli S, Mancebo J, Sirodot M, Hilbert G, Bengler C, Richecoeur J, Gainnier M, Bayle F, Bourdin G, Leray V, Girard R, Baboi L, Ayzac L, PROSEVA Study Group (2013) Prone positioning in severe acute respiratory distress syndrome. N Engl J Med 368:2159-2168

2. Washko GR, O'Donnell CR, Loring SH (2006) Volume-related and volumeindependent effects of posture on esophageal and transpulmonary pressures in healthy subjects. J Appl Physiol (1985) 100:753-758

3. Kumaresan A, Gerber R, Mueller A, Loring SH, Talmor D (2018) Effects of prone positioning on transpulmonary pressures and end-expiratory volumes in patients without lung disease. Anesthesiology 128:1187-1192

4. Mezidi M, Parrilla FJ, Yonis H, Riad Z, Bohm SH, Waldmann AD, Richard JC, Lissonde F, Tapponnier R, Baboi L, Mancebo J, Guerin C (2018) Effects of positive end-expiratory pressure strategy in supine and prone position on lung and chest wall mechanics in acute respiratory distress syndrome. Ann Intensive Care 8:86

5. Riad Z, Mezidi M, Subtil F, Louis B, Guerin C (2018) Short-term effects of the prone positioning maneuver on lung and chest wall mechanics in patients with acute respiratory distress syndrome. Am J Respir Crit Care Med 197:1355-1358 